\title{
Point-of-care three-dimensional printing for craniomaxillofacial trauma
}

\author{
Jeffrey S. Marschall, George M. Kushner \\ Department of Oral and Maxillofacial Surgery, University of Louisville, School of Dentistry, Louisville, KY 40202, USA. \\ Correspondence to: Dr. Jeffrey S. Marschall, Department of Oral and Maxillofacial Surgery, University of Louisville, School of \\ Dentistry, 501 S Preston Street Louisville, KY 40202, USA. E-mail: jsmars03@louisville.edu
}

How to cite this article: Marschall JS, Kushner GM. Point-of-care three-dimensional printing for craniomaxillofacial trauma. Plast Aesthet Res 2021;8:28. https://dx.doi.org/10.20517/2347-9264.2020.222

Received: 20 Dec 2020 First Decision: 4 Feb 2021 Revised: 18 Apr 2021 Accepted: 7 May 2021 Published: 23 Jun 2021

Academic Editors: Pedro Infante-Cossio, Raúl González-García Copy Editor: Yue-Yue Zhang Production Editor: Yue-Yue Zhang

\begin{abstract}
Computer-aided design, three-dimensional printing, and additive manufacturing are revolutionizing craniomaxillofacial trauma surgery. Traditionally, this is completed via third-party vendors during online web meetings. Although this is effective, it can take several weeks to have custom plates arrive, negating its use in acute facial trauma. The price of 3D printers and software needed to complete this in-house are decreasing. This allows for expedited turn around, facilitating treatment in the acute setting. This article serves as a review of fundamental $3 D$ printing principles and describes the process of virtually reducing facial fractures, 3D printing the reduced models, and having a plate ready for surgery in hours.
\end{abstract}

Keywords: 3D printing, facial trauma, computer-aided design and manufacturing

\section{INTRODUCTION}

In general, $3 \mathrm{D}$ printing is a process where material such as a photopolymer resin is cured via ultraviolet laser, layer by layer until the object is complete. However, in the medical field, several steps must be completed before a model can be generated. First, a scan must be completed to capture the anatomical region of interest. In oral and maxillofacial surgery, this is completed using traditional medical grade fan computed tomography (CT) scanners or in-office cone beam scanners of the maxillofacial region. The 
image from these scanners is saved as a standard format called Digital Imaging and Communications in Medicine (DICOM) files. Then, a computer-aided design and manufacturing (CAD/CAM) software package is used to create a model from these images. The model is saved in a file format called standard tessellation language (STL). This file is sent to the $3 \mathrm{D}$ printer for fabrication. Many institutions and individual hospital services are investing in clinic-based $3 \mathrm{D}$ printing labs ${ }^{[1,2]}$ [Figure 1].

Even though 3D printing was first developed in the 1980s, there has been a slow progression to widespread use within craniomaxillofacial surgery. Indeed, craniomaxillofacial surgery was one of the first surgical specialties to adopt this technology. In general, 3D printing can be classified by the technique/method used to build the model/object that is desired. The different classifications of $3 \mathrm{D}$ printers include stereolithography (SLA), selective laser sintering (SLS), fused deposition modeling (FDM), laminated object manufacturing, and electron beam melting. The consumable materials used vary widely, ranging from photopolymerizing resin in SLA printers to titanium powder in SLS machines. In general, SLA printers are considered to provide great accuracy for anatomic models and the resin provides excellent surface finish ${ }^{[3,4]}$. Although there is variability from resin manufactures, one can expect a resin-based SLA model to be slightly brittle and light ${ }^{[3-5]}$. These features are a few of the reasons SLA 3D printers have been popular in medical model manufacturing.

$3 \mathrm{D}$ printing in craniomaxillofacial surgery can range from printing biomimetic scaffolds to aid in tissue engineering to printing models that replicant patient specific anatomy. Since many craniomaxillofacial surgeries are aimed at cutting, recontouring, or reconstructing bone, patient specific models offer tangible objects that give surgeons more data for formulating treatment plans. The current status quo has been used to fabricate patient specific models, surgical/cutting guides, splints, and implants. Anatomic models are likely the most common product that utilizes 3D printing in CMF surgery to date. These models can be used for a variety of reasons. For example, a model can be printed of a mandible that is used for prebending a reconstruction plate before going to the operating room. To this point, if a proposed site of mandible is to be resected, then the contralateral native mandible is mirrored. Furthermore, 3D printed models have been used for contouring orbital reconstruction hardware, often times by mirroring the noninjured side $\mathrm{e}^{[--8]}$. Another object manufactured via 3D printing in CMF surgery is custom patient specific guides. Guides are constructed using the negative space surrounding the patient's anatomy. Guides, albeit a generic term, can include cutting guides around pathology and those used for osteotomies in orthognathic surgery ${ }^{[9,10]}$. Specifically, in craniofacial surgery, operating time is reduced and improved results are obtained when models used for simulation and surgical guides are constructed ${ }^{[11]}$. Lastly, surgical splints can be fabricated. This is most commonly completed using patient's dentition in orthognathic surgery. The splints are negative space models based off of a virtually planned final position of the dentition post-surgical movement. This can be completed in house using software such as Dolphin (Dolphin Imaging, Chatsworth, CA, USA) or via third-party companies.

Repair of patients with fractures of the craniofacial complex is centered on the ability to establish correct pre-morbid occlusion via maxillomandibular fixation when the dentition is present. When the teeth are brought into the correct relationship, the bones generally follow. This allows for open-reduction and internal fixation (ORIF) using titanium bone reconstruction plates. While traditional ORIF has stood the test of time, CAD/CAM principles are changing the way surgery is completed ${ }^{[12-15]}$. CAD/CAM gives craniomaxillofacial surgeons the opportunity to preplan the surgery before going to the operating room. This essentially gives surgeons a blueprint for the surgery. Many surgeons are now using these CAD/CAM principles as their go to method for corrective jaw surgery, microvascular surgery, and craniofacial surgery ${ }^{[16-18]}$. Operating room costs are extremely high; pre-surgery planning has the ability to reduce costs 


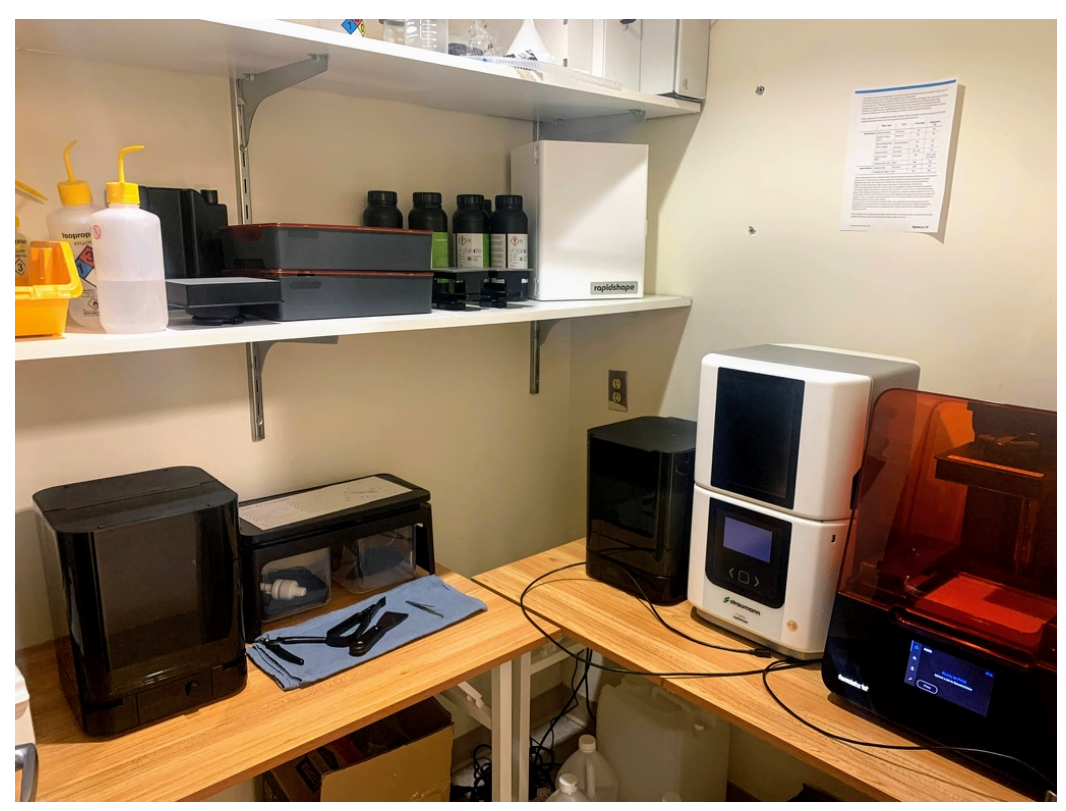

Figure 1. 3D printer lab. An example of a clinic-based 3D printing lab demonstrating several different printers and post-print-processing equipment.

in the operating room. However, considering the timeframe in which acute trauma is ideally treated, there has been less widespread adoption of CAD/CAM principles and/or 3D printing applied to acute facial trauma.

\section{Off-site CAD/CAM vs. point-of-care 3D printing}

Most of the literature discussing CAD/CAM principles and 3D printing for the treatment of acute facial trauma is related to orbital trauma ${ }^{[19]}$. This was an excellent starting point because of the predictability of model fabrication. That is, a mirror image of a non-injured facial skeleton serves as an excellent template to build upon. It was not until recently, however, that studies started to emerge demonstrating the use of CAD/CAM principles with custom milled and/or pre-bent plates for mandibular or complex facial trauma ${ }^{[14,20-24]}$. Currently, these investigations generally follow two distinct paths. A surgeon can either complete the workflow via a third party (i.e., outsource) or choose to keep the process in-house. Each method has its own pro and cons.

First, we consider the outsourced, third-party workflow [Table 1]. This was excellently detailed by Kokosis et al ${ }^{[14]}$ in 2018 in a series of five cases. When a patient is injured, the CT scan used to diagnose the injuries must be uploaded to the portal of the company providing the VSP services. This is surgeon and/or institution dependent. Examples include Synthes ProPlan CMF, KLS Martin IPS, and Stryker 3D Systems VSP. Once the DICOM data (i.e., files from the CT scan) are uploaded, the images and files are reviewed by clinical engineers using the company's proprietary software. The data are then processed, i.e., the CT images are segmented. Simply put, this means the areas of interest from the CT data are isolated. For example, the mandible could be segmented from the rest of craniofacial skeleton to make data processing easier. At the surgeon's convenience, a "go-to-meeting" online VSP session is completed with the clinical engineers. During this meeting, manipulation of the fractures occurs, and a decision is made upon the design of a custom reconstruction bone plate. The third-party clinical engineers check the manufacturability of the custom reconstruction plate. Once the internal checks have been completed by the third party, the virtual case plan is finalized by the clinical engineer and an approval is sent to the surgeon. Once the surgeon 
Table 1. Timeframe for off-site 3D printing

\begin{tabular}{ll}
\hline & Outsourced CAD/CAM and custom plate time frame \\
\hline Day 0 & Patient arrival in ER, CT scan completed \\
Day 1 & CT requested and upload to 3rd party \\
Day 2 & VSP planning session \\
Day 3 & Case finalized; plate starts fabrication \\
Day 4 & Plate and models shipped \\
Day 5 & Plate in transit \\
Day 6 & Plate arrival, sterilized overnight \\
Day 7 & Plate ready for surgery \\
\hline
\end{tabular}

There is variability in this timeframe and it is highly dependent on shipping and scheduling. VSP: virtual surgical planning; ER: emergency room.

approves the plan, the custom reconstruction plate is milled from a titanium block. Once the plate fabrication is completed, it is shipped to the surgeon. The first major advantage of this workflow is the ability to have a custom-milled reconstruction plate. These plates have excellent adaptation and require no bending. The second advantage is the time of the surgeon is engaged in the workflow only during the VSP session and then the surgery. The disadvantage of this workflow is the amount of lead time required to complete all the required steps for plate fabrication and then, obviously, the amount of time required for manufacturing and shipment. This workflow from start to finish is approximately 6-7 days ${ }^{[14]}$. In the authors' experience, this timeline may not be available to all surgeons. One could expect a timeframe of 1014 days from date of injury to plate arrival, assuming the CT scan is uploaded soon after initial completion. This timeframe could be applicable to patients who are admitted, such as a poly-trauma patient with concurrent mandibular or craniofacial trauma. Many of these patients have overriding issues that make this 1-2-week timeframe feasible. However, in many, and indeed often the most acute facial trauma cases, the 12 -week timeframe is less than ideal.

Considering the price of $3 \mathrm{D}$ printers is continuing to drop, for example, the MSRP of a FormLabs Form3 printer is approximately $\$ 3000$, and the learning curve of the CAD/CAM programs is becoming flatter, many surgeons and institutions are turning to point-of-care (POC) manufacturing ${ }^{[25]}$. POC manufacturing refers to the "just-in-time" creation of anatomic models or other items at the place of care, such as the hospital or clinic ${ }^{[25]}$. POC or in-house 3D printing and manufacturing provide cost-effective CAD/CAM technologies in the delivery of services that otherwise would not be feasible due to, at least in part, the high cost of third-party VSP sessions and manufactured products and the lead time required for these services/products ${ }^{[25]}$. Several great examples of surgeon-led in-house manufacturing techniques are starting to emerge within the literature ${ }^{[21-24,26]}$. Furthermore, in-house 3D printing has been demonstrated to decrease operative time ${ }^{[22]}$.

The workflow established by the authors at the University of Louisville (Louisville, KY, USA) is described below [Figure 2]. This workflow is designed for expedited in-house CAD/CAM methods to fit the ideal treatment timeframe for acute craniomaxillofacial trauma ${ }^{[24]}$. This technique merges the benefits of having an online VSP session with on-site 3D model fabrication for preoperative plate bending to virtually reduced mandible/craniomaxillofacial fractures. The first step is completed via image acquisition for diagnosis of the facial injuries. Ideally, this would be obtained using a fan beam medical grade CT scanner with 1.0-mm slices. After the initial surgical consultation, the DICOM files from the CT scanner are transferred to a compact disk. This allows for export to other computers. Ideally, cloud-based file sharing would be the most efficient process. The DICOM files are imported into the CAD software of choice. Our institution utilizes 


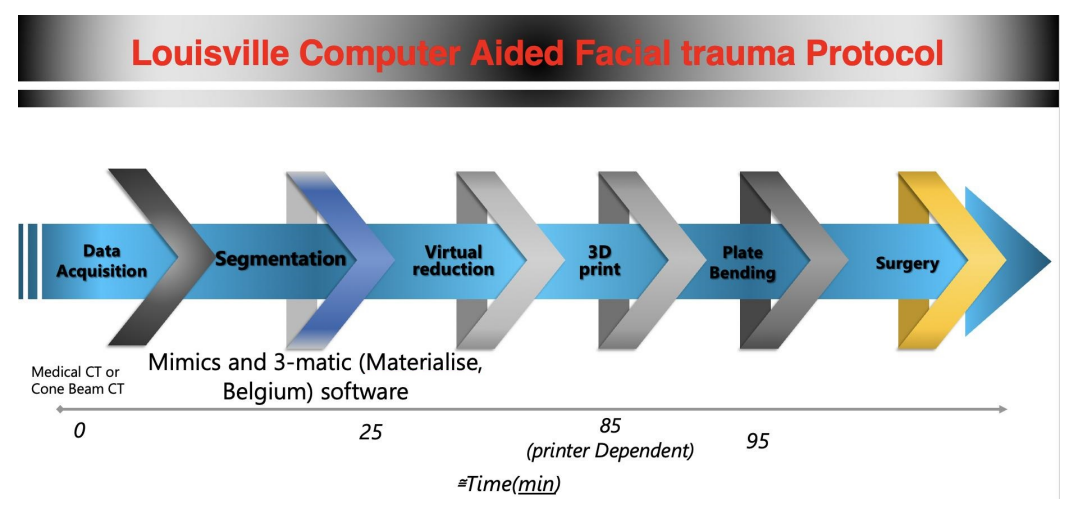

Figure 2. Louisville computer-aided facial trauma protocol.

Mimics (Materialise, Leuven, Belgium) software. This is part of the Materialise innovation suite. Once the files are uploaded into the CAD software, segmentation of the data must be completed (see Marschall et al. ${ }^{[24]}$ for complete details). Segmentation is an essential process for image/data processing and many programs are available. Other examples include Anatomage MD design studio and $\mathrm{D} 2 \mathrm{P}$ from 3D systems. Regardless of the CAD software utilized, the surgeon/institution much ensure they are FDA approved. An example of a free open-source software that is not FDA approved is $3 \mathrm{D}$ slicer. Once the segmentation of the mandible and each fracture is complete, virtual reduction is completed. In the authors' protocol, this is completed via "virtual maxillomandibular fixation". The teeth, and thus the fractured segments, are virtually placed into occlusion, which generally brings the bony segments into their correct pre-morbid position. Once this is completed, the condyles are examined to ensure they are appropriately placed within the Glenoid fossa. This is generally enough to rebuild the fractured mandible/facial skeleton in the computer. This may appear to be a technically challenging maneuver, but, in our experience, it takes a novice software user three runs through the workflow to gain competence. Once the operator is comfortable with this process, segmentation and virtual reduction can be completed within 10-35 min depending on the operator.

After virtual reduction is completed, the new mandible is exported as a STL file which can be read by the 3D printing software. The printing software is dependent on the $3 \mathrm{D}$ printer used by the clinician. FormLabs printers use pre-form. Considering many $3 \mathrm{D}$ printers print using a platform that moves vertically out of a resin reservoir, supports must be added to the object, which is done in the software. Supports are important for $3 \mathrm{D}$ printing as they hold the model in place through the entire printing process. Although a clinician can use $3 \mathrm{D}$ printers that have slightly different working principles (SLA, FDM, DLP, etc.), that discussion is beyond the scope of this review. Once the object has been supported appropriately, printing can begin. The speed of printing is highly variable. For example, an inexpensive FormLabs (Somerville, MA) Form3 printer may take 5-6 h to print an entire mandible depending on resolution, while the NewPro (NewPro 3D Vancouver, British Columbia, Canada) NP1 can print an entire mandible in approximately $1 \mathrm{~h}$. We use both of these printers in our workflow depending on the timeframe required. Once the print is completed, the model must be washed in isopropyl alcohol for approximately $15 \mathrm{~min}$ and then cured for $1 \mathrm{~h}$.

Once the virtually reduced mandible has finished the curing process, it can be used as a template for reconstruction plate preoperative adaptation. There are several major advantages of preoperative plate adaptation. First, plate bending occurs out of the operating room, which can be as expensive as $\$ 100$ per minute $^{[22]}$. Second, the plate bending can occur leisurely in the office or even at home. Last, the printed mandible can be held in the surgeon's hand and examined in $3 \mathrm{D}$. This model is not bound by soft tissues 
and visibility is not obstructed by bleeding or hemorrhage; this facilitates a truly unobstructed view. The fracture can be examined from aspects that cannot be truly appreciated during the operation or from reviewing the CT scan. Once the plate has been bent, it can be sterilized before surgery. Our current clinical experience demonstrated that most plates can be adapted in between 7 and 20 min when using this workflow. Obviously, the fractures can be approached via any method the surgeon chooses. The addition of a pre-bent plate, however, may make fractures that are difficult to treat via an intra oral approach more predictable, potentially saving the patient a cutaneous scar.

When comparing these two methods, one must also consider cost. Potentially high up-front costs are associated with the POC/in-house method. However, this is dependent on the equipment purchased. $3 \mathrm{D}$ printers can be as little as several hundred US dollars or hundreds of thousands of dollars. There is also variability in software costs. However, once these items have been purchased, the fixed costs of consumables are generally rather low. For example, a 3D-printed mandible using a resin-based SLA printer (i.e., FormLabs) costs approximately \$7.00. Direct cost comparisons between in-house and outsourced methods are difficult to complete. Furthermore, depending on the contractual agreements between the hospital and company providing the CAD/CAM services, custom-milled plate costs can be different. Custom-milled plates can cost as much as $\$ 5000-\$ 6000$ (nearly double the costs of a Form3 printer). Added to this are the costs associated with model fabrication, planning session, etc. In our experience, the all-inclusive costs of a VSP, model fabrication, and custom-milled plate approach $\$ 10,000$. When compared to a POC approach, stock reconstruction plates are approximately $\$ 1000$. In the future, these techniques can be trained so that in-house technicians can complete the virtual reduction, decreasing surgeon time. Furthermore, in-house custom-milled plates can be fabricated in this same expedited manner.

\section{In-house CAD/CAM case report}

A 48-year-old male was assaulted causing bilateral mandibular fractures [Figure 3]. To assess the extent of his injuries, a standard maxillofacial CT scan using $1.5-\mathrm{mm}$ cuts was completed in our university's emergency department, whereupon the facial trauma service on call was consulted (it should be noted 1.0-mm cuts are preferred). After the consultation was complete, the DICOM files from the CT scanner were transferred to a compact disk and loaded onto our CAD/CAM computer within our 3D printing lab. The patient's occlusion was reestablished virtually to aid in reduction of the fractures, essentially a virtual MMF. The mandible was then printed according to the protocol described above ${ }^{[24]}$. Two locking reconstruction plates were preoperatively adapted to the $3 \mathrm{D}$ printed mandible. Once the patient was taken to the OR for definitive reconstruction, the fractures were all exposed in standard fashion. The mandibular bone was reduced, and the pre-bent mandibular reconstruction plates were applied with near perfect adaptation. The incisions were closed in standard fashion and a postoperative CT scan was ordered. The final result obtained was overlaid to our preoperative virtual plan to assess how accurately we executed the surgery using Geomagic Design X (3D Systems, Rock Hill, SC, USA). A root mean square value of 1.9 was obtained, with the highest variation occurring in sites where the reconstruction plate was applied. The patient went on to heal uneventfully.

\section{CONCLUSION}

$\mathrm{CAD} / \mathrm{CAM}$ and $3 \mathrm{D}$ printing are changing the way craniomaxillofacial surgeons treat their patients. The evolution of this is slowly making its way through the entire scope of the surgical specialties dedicated to facial differences, pathology, and injuries. When a surgeon is faced with a facial injury that could benefit from CAD/CAM planning, they must choose if they want to complete the workflow in-house or outsource it to a third party. Both have their own pros and cons. In-house techniques may require more surgeon time out of the OR, but, generally speaking, it can have a plate ready for sterilization much more quickly. The 

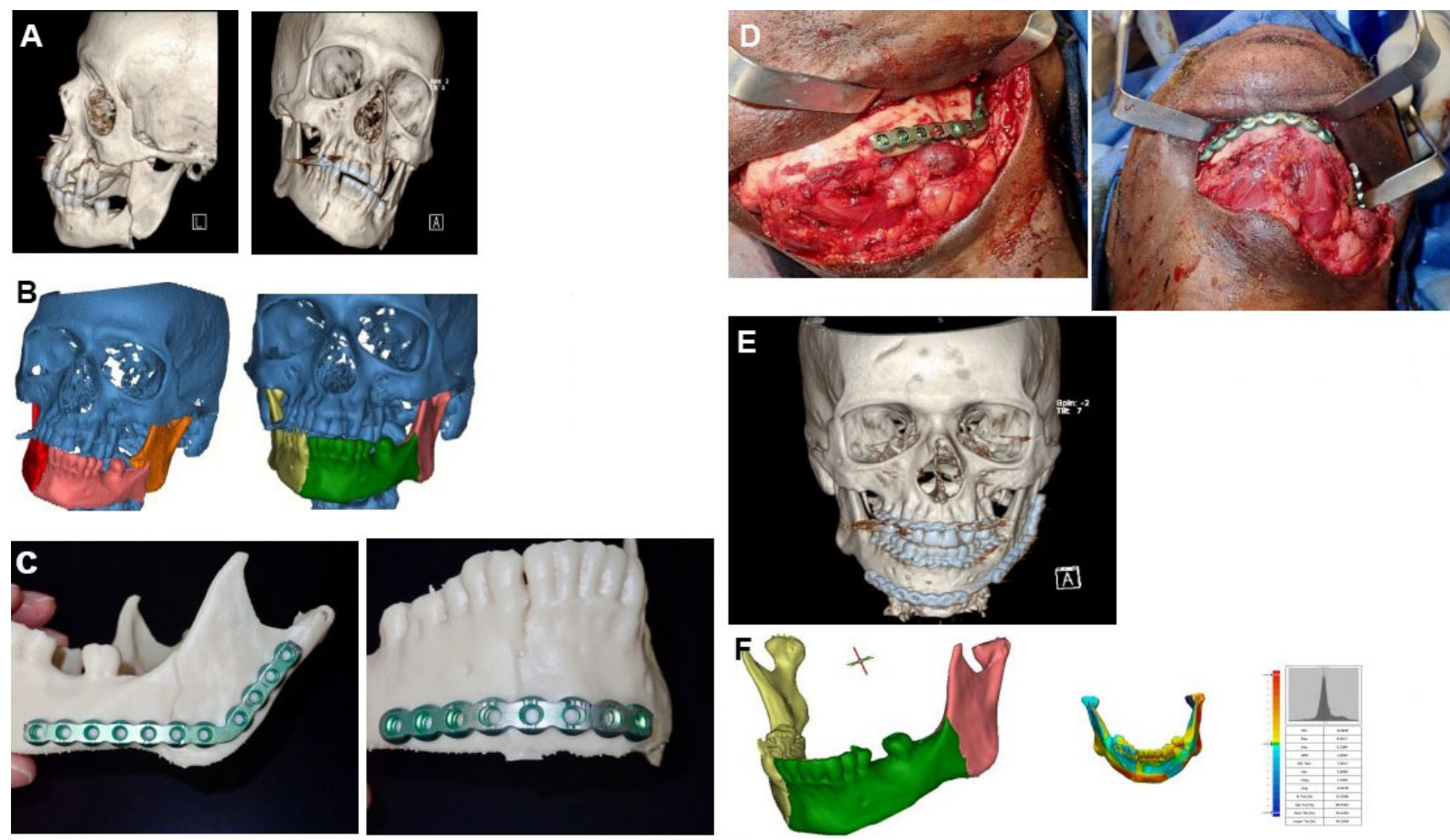

Figure 3. Representative case. A 48-year-old male who suffered mandibular fractures: (A) preoperative CT scan; (B) (Left) segmentation of fractured mandible and (Right) virtual reduction of mandible; (C) 3D-printed reconstructed facial bones with preoperatively bent reconstruction plates; (D) transcervical approach to the mandible to expose both fractures; (E) postoperative CT; and (F) (Left) preoperative plan and (Right) heat map of merged postoperative result and preoperative plan. Areas cold in color (blue and green) represent areas of high accuracy, while areas of hot color (orange and red) represent areas of relative lower accuracy.

learning curve required for using the CAD/CAM software can be considered a major barrier by some. Outsourced workflows have the benefit of decreased surgeon time (the clinical engineer spends the time completing the segmentation and reduction), as well as the ability to produce custom-milled reconstruction plates. However, this takes more time and is substantially more expensive. In our institution, we tend to use our in-house workflow for acute trauma and the outsourced workflow for revision cases (not acute) where a previous plate may have failed and could benefit from the increased strength of a custom-milled plate. Regardless, facial surgeons need to adapt to this new technology, as it may soon become ubiquitous across their practice.

\section{DECLARATIONS}

\section{Authors' contributions}

Wrote and edited the manuscript: Marschall JS, Kushner GM

Availability of data and materials

Not applicable.

\section{Financial support and sponsorship}

None.

\section{Conflicts of interest}

All authors declared that there are no conflicts of interest. 


\section{Ethical approval and consent to participate}

Not applicable.

\section{Consent for publication}

Not applicable.

\section{Copyright}

(C) The Author(s) 2021.

\section{REFERENCES}

1. Ghai S, Sharma Y, Jain N, Satpathy M, Pillai AK. Use of 3-D printing technologies in craniomaxillofacial surgery: a review. Oral Maxillofac Surg 2018;22:249-59. DOI PubMed

2. Nyberg EL, Farris AL, Hung BP, et al. 3D-printing technologies for craniofacial rehabilitation, reconstruction, and regeneration. Ann Biomed Eng 2017;45:45-57. DOI PubMed PMC

3. Chang PS, Parker TH, Patrick CW Jr, Miller MJ. The accuracy of stereolithography in planning craniofacial bone replacement. $J$ Craniofac Surg 2003;14:164-70. DOI PubMed

4. Choi JY, Choi JH, Kim NK, et al. Analysis of errors in medical rapid prototyping models. Int J Oral Maxillofac Surg 2002;31:23-32. DOI PubMed

5. Choi JW, Kim N. Clinical application of three-dimensional printing technology in craniofacial plastic surgery. Arch Plast Surg 2015;42:267-77. DOI PubMed PMC

6. Callahan AB, Campbell AA, Petris C, Kazim M. Low-cost 3D printing orbital implant templates in secondary orbital reconstructions. Ophthalmic Plast Reconstr Surg 2017;33:376-80. DOI PubMed

7. Perry M, Banks P, Richards R, Friedman E, Shaw P. The use of computer-generated three-dimensional models in orbital reconstruction. Br J Oral Maxillofac Surg 1998;36:275-84. DOI PubMed

8. Jacobs CA, Lin AY. A new classification of three-dimensional printing technologies: systematic review of three-dimensional printing for patient-specific craniomaxillofacial surgery. Plast Reconstr Surg 2017;139:1211-20. DOI PubMed

9. Farrell BB. Evolving management of dentofacial deformities with digital planning and patient-specific fixation. Atlas Oral Maxillofac Surg Clin North Am 2020;28:59-71. DOI PubMed

10. Weyh AM, Quimby A, Fernandes RP. Three-dimensional computer-assisted surgical planning and manufacturing in complex mandibular reconstruction. Atlas Oral Maxillofac Surg Clin North Am 2020;28:145-50. DOI PubMed

11. Tack P, Victor J, Gemmel P, Annemans L. 3D-printing techniques in a medical setting: a systematic literature review. BioMed Eng OnLine 2016:15. DOI PubMed PMC

12. Hanasono MM, Skoracki RJ. Computer-assisted design and rapid prototype modeling in microvascular mandible reconstruction. Laryngoscope 2013;123:597-604. DOI PubMed

13. Kim YC, Jeong WS, Park TK, Choi JW, Koh KS, Oh TS. The accuracy of patient specific implant prebented with 3D-printed rapid prototype model for orbital wall reconstruction. J Craniomaxillofac Surg 2017;45:928-36. DOI PubMed

14. Kokosis G, Davidson EH, Pedreira R, Macmillan A, Dorafshar AH. The use of computer-aided design and manufacturing in acute mandibular trauma reconstruction. J Oral Maxillofac Surg 2018;76:1036-43. DOI PubMed

15. Miloro M, Markiewicz MR. Virtual surgical planning for inferior alveolar nerve reconstruction. J Oral Maxillofac Surg 2017;75:24428. DOI PubMed

16. Rodby KA, Turin S, Jacobs RJ, et al. Advances in oncologic head and neck reconstruction: systematic review and future considerations of virtual surgical planning and computer aided design/computer aided modeling. J Plast Reconstr Aesthet Surg 2014;67:1171-85. DOI PubMed

17. Swennen GR, Mollemans W, Schutyser F. Three-dimensional treatment planning of orthognathic surgery in the era of virtual imaging. J Oral Maxillofac Surg 2009;67:2080-92. DOI PubMed

18. Toto JM, Chang EI, Agag R, Devarajan K, Patel SA, Topham NS. Improved operative efficiency of free fibula flap mandible reconstruction with patient-specific, computer-guided preoperative planning. Head Neck 2015;37:1660-4. DOI PubMed

19. Mazzoni S, Bianchi A, Schiariti G, Badiali G, Marchetti C. Computer-aided design and computer-aided manufacturing cutting guides and customized titanium plates are useful in upper maxilla waferless repositioning. J Oral Maxillofac Surg 2015;73:701-7. DOI PubMed

20. Sharkh H, Makhoul N. In-house surgeon-led virtual surgical planning for maxillofacial reconstruction. . J Oral Maxillofac Surg 2020;78:651-60. DOI PubMed

21. Elegbede A, Diaconu SC, McNichols CHL, et al. Office-based three-dimensional printing workflow for craniomaxillofacial fracture repair. J Craniofac Surg 2018;29:e440-4. DOI PubMed

22. King BJ, Park EP, Christensen BJ, Danrad R. On-site 3-dimensional printing and preoperative adaptation decrease operative time for mandibular fracture repair. J Oral Maxillofac Surg 2018;76:1950.e1-8. DOI PubMed

23. Luu K, Pakdel A, Wang E, Prisman E. In house virtual surgery and 3D complex head and neck reconstruction. J Otolaryngol Head Neck Surg 2018;47:75. DOI PubMed PMC

24. Marschall JS, Dutra V, Flint RL, et al. In-house digital workflow for the management of acute mandible fractures. J Oral Maxillofac 
Surg 2019;77:2084.e1-9. DOI PubMed

25. Arce K, Morris JM, Alexander AE, Ettinger KS. Developing a point-of-care manufacturing program for craniomaxillofacial surgery. Atlas Oral Maxillofac Surg Clin North Am 2020;28:165-79. DOI PubMed

26. Williams FC, Hammer DA, Wentland TR, Kim RY. Immediate teeth in fibulas: planning and digital workflow with point-of-care 3D printing. J Oral Maxillofac Surg 2020;78:1320-7. DOI PubMed 